\title{
TOLL-LIKE RECEPTORS AND THEIR CONTRIBUTION TO INNATE IMMUNITY: FOCUS ON TLR4 ACTIVATION BY LIPOPOLYSACCHARIDE
}

\author{
Maciej CZERKIES, Katarzyna KWIATKOWSKA \\ Institute of Experimental Biology, Polish Academy of Sciences, \\ Department of Cell Biology, Warsaw, Poland
}

DOI: $10.2478 /$ acb-2014-0001

Summary: Mechanisms of innate immunity are triggered as a result of recognition of evolutionarily conserved structures of microorganisms, named pathogen-associated molecular patterns. Their recognition is mediated by specialized receptors which initiate signalling cascades leading to expression of pro-inflammatory mediators and regulation of acquired immunity. Among several classes of such receptors, Toll-like receptors (TLRs) are extensively studied as they can sense an array of microbial cell wall and membrane components as well as single- and double-stranded RNA and DNA motifs typical for microorganisms. Each TLR consists of a ligand-binding domain containing leucine-rich repeats, a single transmembrane domain and a signalling TIR domain. After ligand binding, TLRs dimerize which facilitates the interaction of their TIR domains with adaptor proteins triggering signalling cascades. TLRs engage four common adaptor proteins, about ten signalling kinases, and a few transcription factors including NFKB, IRF and AP-1. In this review, special attention is paid to TLR4 activated by lipopolysaccharide (LPS), a component of the outer membrane of Gram-negative bacteria, since an exaggerated response to LPS may lead to potentially deadly septic shock. In recent years considerable progress has been made in the understanding of how the cooperation of several proteins, including CD14, TLR4/MD-2 complex and scavenger receptors, modulates the cell response to LPS. These studies have also revealed a dichotomy of signalling pathways triggered by TLR4 which depends on the participation of MyD88 and TRIF adaptor proteins and leads to the expression of genes encoding pro-inflammatory cytokines and type I interferons, respectively. The key event in the TRIF-dependent pathway is the internalization of activated TLR4.

Key words: Toll-like receptors, signal transduction, lipopolysaccharide, sepsis, innate immunity 


\section{INTRODUCTION}

A key role in the functioning of the vertebrate immune system is played by evolutionarily old mechanisms of innate immunity. These mechanisms allow for a rapid response directed against microbes which have successfully overcome the physical barriers protecting the body. They also participate in the mobilization and control of acquired immunity mechanisms, assuring maintenance of long-term immunological memory.

One of the most significant properties of the immune system is its ability to distinguish „foreign” microbe particles from „own” molecules of the host body. This distinction is important because of the need for early detection of pathogens and for avoiding the risk of developing autoimmune diseases. Another problem which must be confronted by the immune system is the need to recognize a large number of potentially hazardous species belonging to so variable microorganisms as bacteria, viruses, fungi and protozoa. This goal is fulfilled by Pattern Recognition Receptors (PRRs) present in cells such as monocytes, macrophages, neutrophils, dendritic and epithelial cells. These receptors are capable of recognizing evolutionarily conserved molecules typical for entire groups of microbes and absent from the host body. These molecules are called Pathogen-Associated Molecular Patterns (PAMPs) [39].

\section{PATTERN RECOGNITION RECEPTORS (PRRS)}

Typical examples of the molecular patterns recognised by PRRs are components of the bacterial cell wall and, in the case of Gram-negative bacteria, also components of the outer membrane of the bacteria. These structures contain molecules typical for bacteria, such as peptidoglycans, lipopolysaccharides and specific lipoproteins. Another significant group of ligands recognised by PRRs are nucleic acids. In this case their distinction from analogous host molecules is possible due formation of specific structures, the presence of specific sequences and differences in modifications of microbial nucleic acids (e.g., absence of methylated $\mathrm{CpG}$ motifs).

It is postulated that, apart from the recognition of pathogens, PRRs play an additional function by participating in responses to endogenous signals indicating tissue injury. Such signals can involve products of proteolysis of the extracellular matrix, which accumulate during tissue damage, and certain intracellular proteins, such as HMGB-1 (High-Mobility Group Box-1), released during cell apoptosis [84]. Such endogenous molecules capable of activating the immune system with an involvement of PRRs are termed DAMPs (Damage/Danger-Associated Molecular 
Patterns) [3]. Nevertheless, it should be noted that according to some investigators the ability of DAMP molecules to directly activate PRRs is questionable. It is suggested that the endogenous factors just increase the sensitivity of cells to trace amounts of PAMPs [10].

Depending on their mode of functioning PRRs are divided into three classes: secreted receptors, receptors which mediate phagocytosis, and signalling receptors (fig. 1). The former comprise soluble molecules of opsonin type which bind to the surface of pathogens, facilitating their internalization and initiating classical and lectin pathways of complement activation. PRRs engaged in phagocytosis are transmembrane proteins which mediate internalization of pathogens, leading to degradation of the internalized particles and presentation of antigens released from them. The third class of PRRs comprises several receptor families with signalling domains which are capable of activating transcription of genes coding for pro-inflammatory proteins. They include:

- Toll-Like Receptors (TLRs) - the most thoroughly characterized family of PRRs described in greater detail below;

- RIG-I-Like Receptors (RLRs) - a family of three cytoplasmic helicases which detect double-stranded viral DNA. Two of them, RIG-1 and MDA-5, containing CARD (Caspase Activation and Recruitment Domain), induce a signalling pathway leading to the synthesis of type I interferons; the third member of the family, LPG, lacks the CARD domain and functions as a positive regulator of RIG-1 and MDA5 [66]. RLRs have been shown to take part in recognition of viruses belonging to families of Flaviviridae (e.g., hepatitis C virus), Picornaviridae (e.g., Polio virus) and Paramyxoviridae (e.g., Newcastle disease virus);

- NOD-Like Receptors (NLRs) - large family of receptors (23 such receptors have been identified in humans and 34 in mice) which recognize PAMPs in the cytoplasm. The receptors may carry various effector domains, the most typical of which include CARD, PYD (Pyrin Domain) and BIR (Baculovirus Inhibitor Repeats) domains. The numerous ligands recognized by the receptors include peptidoglycan, its component $\gamma$-D-glutamyl-meso-diaminopimelic acid, muramyl dipeptide from the cell wall of Gram-positive bacteria, flagellin, and compounds released due to cell damage (e.g., uric acid) and certain bacterial toxins. Activation of these receptors can lead to formation of a multimolecular complex termed the inflammasome, responsible for promotion of inflammatory processes and capable of initiating a specific form of apoptosis, pyroptosis [4, 39];

- cytosolic receptors of DNA - a group of mostly unidentified proteins, responsible for initiation of production of type I interferons in response to plasmid, viral or bacterial DNA in the cytoplasm. It is postulated that the process progresses with an involvement of DAI, STING and ATG9a proteins [39]. 


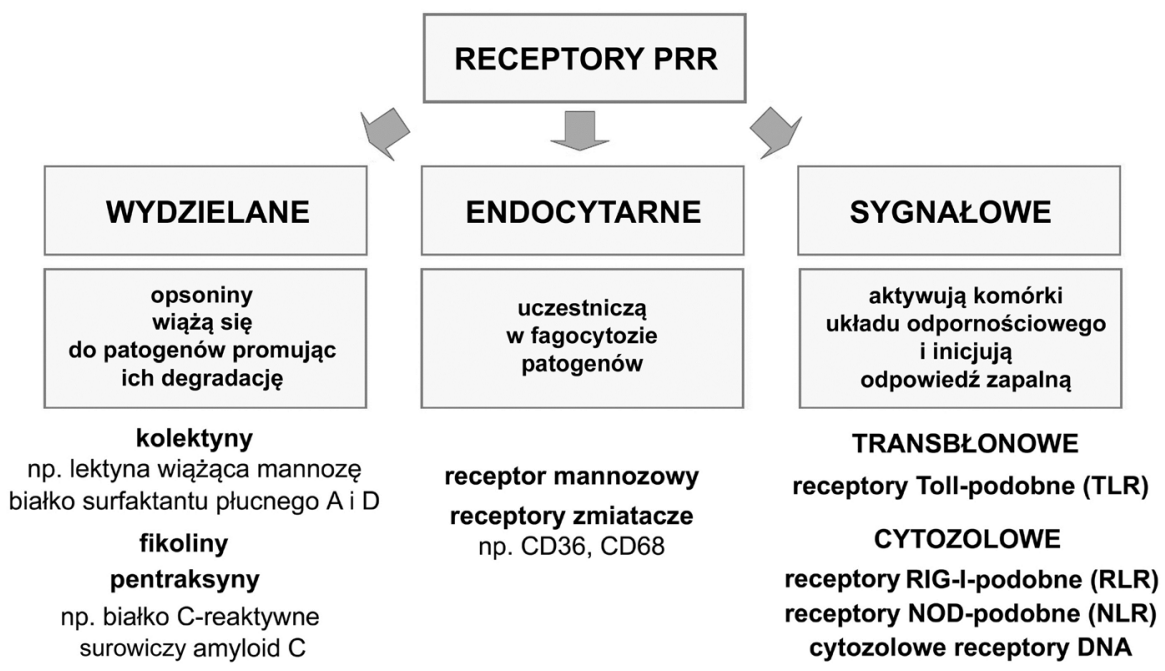

FIGURE 1. Classification of pattern recognizing receptors depending on their function. The receptors mediating phagocytosis are located in the plasma membrane of phagocytic cells, such as macrophages and dendritic cells. TLRs are present also in other cells of immune system, as well as in epithelial and endothelial cells, and in fibroblasts. RLRs and some NLRs (e.g., NOD1) are present in the cytoplasm of most cell types

It is worth noting that despite the significant structural variability of both the ligands and the PRRs binding them, the scheme of the inflammatory response activation and its course remain similar. Typical for PRRs is the effect of narrowing the numbers of different molecules engaged in cell activation, starting at a few hundreds types of recognised PAMPs, through few tens of receptors and over ten adaptor proteins linked to them, to just about ten main kinases engaged in signal transduction and several transcription factors controlling large groups of genes. The main kinases involved in PRR signalling include those belonging to the IRAK (Interleukin-1 Receptor-Associated Kinase), TRAF (TNF Receptor-Associated Factor), MAPK (Mitogen-Activated Protein Kinase) and IKK (Iқ̧B Kinase) families.

Finally, the signalling pathways triggered by TLRs, RLRs and NLRs lead to activation of just a few main transcription factors: $\mathrm{NFқB} \mathrm{(Nuclear} \mathrm{Factor} k$-light chain-enhancer of activated B cells), AP-1 (Activator Protein-1) and IRF 3, 7 and 5 (Interferon Regulatory Factors). The first two mobilize expression of hundreds of genes responsible for production of cytokines, chemokines and pro-inflammatory enzymes. The most important compounds of this type include tumour necrosis factor $\alpha$ (TNF- $\alpha$ ), interleukins IL-1, $-6,-8,-10,-15$ and -18 , and chemokines MIP-1, MCP1, 2 and 3. Transcription factors IRF3 and IRF7 regulate expression of genes coding for type I interferons, IFN- $\alpha$ and IFN- $\beta$. 
The main effect of expression of genes under control of these transcription factors is initiation and regulation of inflammatory responses as well as activation of the complement system, stimulation of phagocytes' activity, and apoptosis of infected cells. Moreover, the role of PRRs in activation and control of acquired immune responses has become increasingly better understood the recent decade [24, 67]. Activation of PRRs is known to positively affect expression of genes encoding molecules of the main histocompatibility complex and co-stimulatory molecules, such as CD80 and CD86, eventually promoting activation of T helper cells. On the other hand, cytokines released by cells in response to activation of PRRs induce differentiation of $\mathrm{T}$ lymphocytes into one of the subpopulations, e.g., Th1 or Th2, determining the subsequent immune reactions [46]. For example, activation of TLR4 stimulates secretion of interleukins, including IL-1, IL-12 and IL-18, promoting development of Th1-type response [67]. Activation of TLRs may also modulate the course of immune reactions through their effect on the functioning of regulatory cells, such as lymphocytes Treg CD4+ CD25+FoxP3+ [16].

\section{TOLL-LIKE RECEPTORS (TLRS)}

\section{GENERAL CHARACTERISTICS}

TLRs form a family of transmembrane proteins with a similar, three-domain structure and high homology to the Toll protein of Drosophila melanogaster. "Toll" in German means „fantastic”, „mad” and was used for the first time by Cristina Nüsslein-Volhard and Kathryn Anderson to denote a gene whose mutation in mother leads to a disturbed development of Drosophila embryo along its dorso-ventral axis [1]. Subsequent studies in the Jules A. Hoffmann's group demonstrated that the Toll protein is of key importance for the resistance of the fruit fly to fungal infections [41]. A protein of the Toll family was linked for the first time with vertebrate immunity at the end of 1990 s owing to studies conducted, among others, by the Bruce Beutler's team [48, 59]. For their discoveries on the activation of innate immunity J.A. Hoffmann and B. Beutler received the Nobel prize in medicine or physiology for 2011. Subsequent screening of sequenced vertebrate genomes has led to the identification of about 100 TLR genes. Genes encoding TLRs have been also detected in the genomes of invertebrates as diverse as the nematode Caenorhabditis elegans, the horseshoe crab Tachypleus tridentatus, and the sea urchin Strongylocentrotus purpuratus. Since proteins structurally and functionally similar to TLRs have been also detected in plants, these receptors seem to represent one of the most ancient component of the host defence mechanisms $[42,14]$. 
Until now, thirteen TLRs have been identified and described in mammals. Twelve of them are expressed in mice and ten in humans. They were grouped into six families on the basis of amino acid sequence homology (fig. 2). In general, each subfamily of the receptors recognize one vast class of PAMPs and most of vertebrates carry at least one receptor of each subfamily. TLRs are present in cells of the immune system, epithelial cells of the intestines (TLR4 and 5), urinary bladder, lungs, liver and kidneys (TLR11). Recently, receptors of the TLR11-13 group were detected in cells of the mammalian central nervous system [64, 50]. In humans the entire TLR11-13 subfamily is represented only by a pseudogene, which is supposed to condition the sensitivity of humans to infections with uropathogenic strains of Escherichia coli. In mice, on the other hand, the gene coding for TLR10 is inactive $[18,86]$.

TLRs can also be classified into two groups based on their location within a cell (fig.2). Receptors of subfamilies TLR1 (TLR1, 2, 6 and 10), TLR4 and TLR5 subfamilies are present mainly in the plasma membrane. Due to their localization on

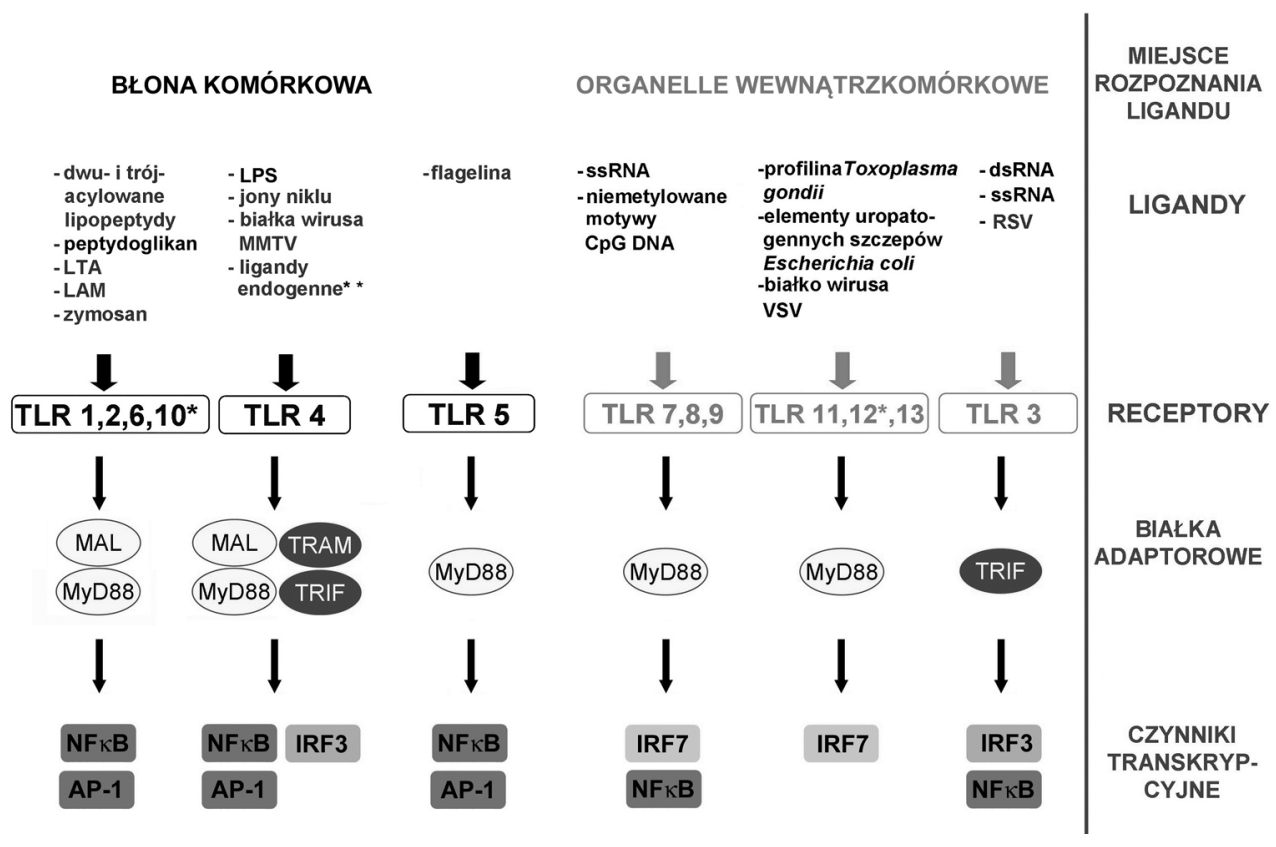

FIGURE 2. Classification and properties of mammalian TLRs. *Ligands of TLR10 and TLR12 have not yet been identified. **Some studies indicate that TLR4 and TLR 2 are able to bind endogenous molecules generated upon tissue and cell damage. A putative list of such molecules includes heat shock proteins, nuclear proteins and components of the extracellular matrix [3]. Other studies argue against this possibility and posit that endogenous molecules can increase sensitivity of cells toward minute amounts of PAMPs [10]. LTA - lipoteichoic acid, LAM - lipoarabinomannan, RSV - respiratory syncytial virus, MMTV - mouse mammary tumour virus, VSV - vesicular stomatitis virus, ssRNA single-stranded viral RNA, dsRNA - double-stranded viral RNA. Based on [34, 39] 
the cell surface they recognize elements of the cell walls, capsules and motility apparatus of pathogenic microbes. On the other hand, receptors of subfamilies TLR3, TLR7-9 and TLR11-13 are located in intracellular organelles, such as endosomes, and take part mainly in the detection of nucleic acids of pathogens. Until recently, TLR11 was thought to be a surface receptor but further studies have confirmed its intracellular localization [58].

Individual TLR subfamilies also differ between each other in their preference for adaptor proteins, which interact with the receptors and allow for generation of signalling pathways (fig.2). Until now, five adaptor proteins used by TLRs have been identified: MyD88 (Myeloid Differentiation primary response gene 88), TIRAP/Mal (TIR domain-containing Adaptor Protein/MyD88-adaptor-like), TRIF/ TICAM-1 (TIR domain-containing adaptor inducing IFN- $\beta / T I R$-Containing Adaptor Molecule 1), TRAM/TICAM2 (TRIF-Related Adaptor Molecule/TIR-Containing Adaptor Molecule 2) and SARM (Sterile $\alpha$ - and HEAT/armadillo motif-containing protein), of which the first four participate in various combinations in signal generation while SARM is a negative regulator of the TRIF-dependent pathway $[77,5]$. Except TLR3, most TLRs mobilize signalling pathways with participation of MyD88, while TRIF is involved in signalling by TLR3 and TLR4 only [77, 34]. The binding of distinct adaptor proteins by TLRs leads to the generation of distinct signalling pathways, which provides one of the mechanisms allowing a better adjustment of the cellular response to the arising danger.

\section{STRUCTURE OF TLRS}

TLRs are type I glycoproteins with a single transmembrane helix which links the $\mathrm{N}$-terminal domain responsible for ligand binding with the signalling domain at the $\mathrm{C}$-terminus of the receptor (fig. 3A). Analysis of the amino acid sequences and crystal structures of several TLRs pointed to a similar, evolutionarily conserved scheme $[7,27,36,45,57]$. The domain located at the N-terminus of the receptors is usually termed "ectodomain" but it should be remembered that in cases of intracellular receptors the domain is directed toward the lumen of the organelles. In all TLRs this domain consists of 16-28 leucine-rich repeats (LRRs) [47] (Fig. 3B). One of the best characterized is TLR4 receptor, activated by lipopolysaccharide (LPS), which contains 22 such motifs. The LRR motif consists of 20-30 amino acids of an evolutionarily conserved sequence LxxLxLxxN (where L represents leucine, $\mathrm{N}$ - asparagine, $\mathrm{x}$ - any amino acid) and a variable region. The extracellular domain, containing a few to several dozens of LRR motifs, has been detected in over 6000 proteins. For around 50 of these proteins, including a few TLRs, their crystal structures has been solved: all of them have a similar horseshoe-like shape (Fig.3A). The LxxLxLxxN sequences are located on the concave side of the horseshoe-like struc- 


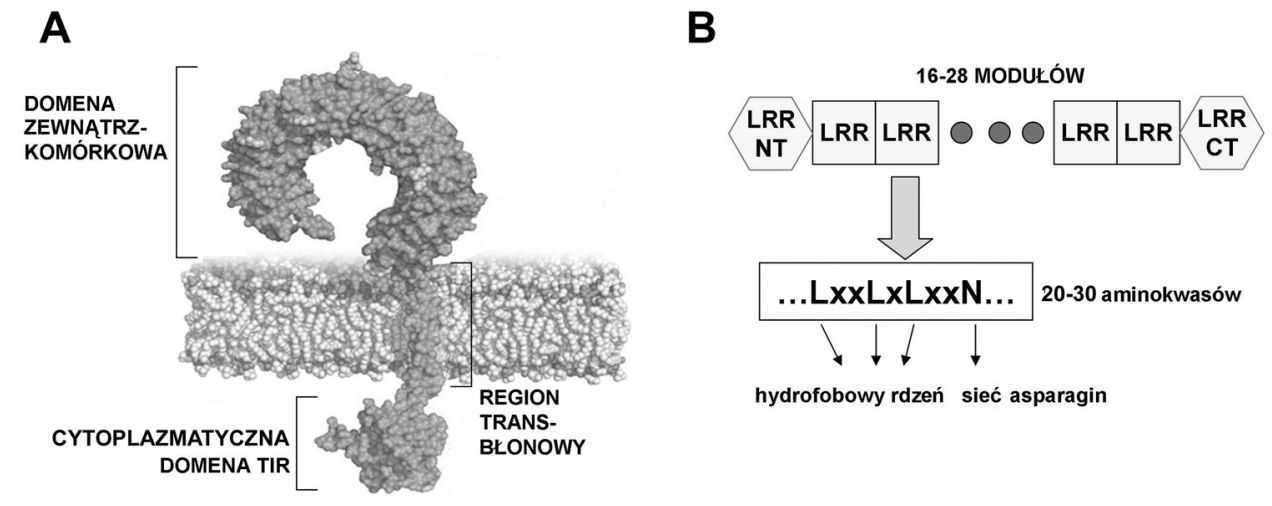

B

FIGURE 3. Structure of Toll-like receptors. A - Model of TLR monomer in the plasma membrane exemplified by TLR3 (acc. to [45], modified). B - Extracellular domain of TLRs containing leucine -rich repeats LRR (acc. to [31], modified). The LxxLxLxxN sequences form parallel $\beta$-strands building the concave part of the domain. The convex part consists of variable residues of the LRR motifs (not shown) which form helices and loops

ture within the parallel $\beta$-strands that form it; the variable regions of LRRs, on the other hand, form the outer, exposed surface of the horseshoe and can play significant functions in interactions with ligands. The leucine residues together with the other hydrophobic amino acids of LRR motifs form a continuous hydrophobic core of the TLR ectodomain. The domain structure is additionally stabilized by numerous hydrogen bonds of the asparagine residues of the LRR motifs [26, 38].

Ectodomains of several TLRs conform to such a „typical” structure of LRR motif-containing proteins. In the case of TLR4, however, and also the entire TLR1 subfamily, the amino acid sequences of the LRR motifs in the central portion of the ectodomain diverge from the evolutionarily conserved pattern: the motifs lack the typical asparagine network which stabilizes their structure, and moreover, they manifest variability also in the number of amino acids forming the motifs. The differences in the structure of the LRR motifs result in the fragmenting of the receptor ectodomain into three subdomains and in the exposure of inner pockets of a hydrophobic character $[27,32,36]$. At present, the structural divergences are postulated to play a role in adaptation of the receptors to binding of ligands of a specific chemical character. Receptors with a typical structure, such as TLR3 and TLR7-9, bind hydrophilic ligands, such as nucleic acids, using the outer surface of the ectodomain. On the other hand, receptors with the distinct three subdomains are capable of binding hydrophobic ligands using the exposed inner hydrophobic pockets. In such a way TLR1, 2 and 6 recognize bi- and tri-acylated bacterial lipopeptides and lipoproteins [32]. TLR4 is the only receptor of the TLR family that does not bind its ligand LPS directly but requires an assistance of a co-receptor, MD-2 protein, for 
the binding $[35,52,55]$. The hydrophobic pockets present in TLR4 ectodomain are thought to allow for dimerization of the receptor through binding of one of the six lipid chains in LPS by one of the receptors while the remaining lipid chains of LPS remain buried in a hydrophobic pocket of MD-2 protein associated with a neighbouring TLR4 molecule [61].

It should also be added that TLRs, similarly to other proteins containing LRR motifs, carries at their amino- and carboxyl-termini so called LRRNT and LRRCT domains (fig. 3B). These domains contain no LRR motifs but include cysteine residues forming disulphide bonds, in this way additionally stabilizing the structure of the ectodomain and protecting its hydrophobic core from exposure to the environment. Another feature of TLRs is the presence of evolutionarily conserved amino acid sequences in the extracellular domain (mainly on its surface), where N-glycosylation takes place. This modification is believed to be important for ligand recognition and localization and transport of the receptors [70, 78].

The cytoplasmic domain of TLRs, responsible for initiation of signal generation, is the TIR (Toll/IL-11receptor homology) domain, also present in receptors for interleukins and in adaptor proteins of TLRs; due to its presence these three protein families are considered to form a single superfamily. The TIR domain contains around 200 amino acid residues. They form a centrally located $\beta$ sheet of five strands $\beta \mathrm{A}-\beta \mathrm{E}$, surrounded by five helices, $\alpha \mathrm{A}-\alpha \mathrm{E}$. Loops which link the elements protrude over the domain surface and most probably play a role in its multimerization required for the formation of a signalling complex [54, 81].

Analysis of the crystal structures of several TLRs from various subfamilies in complexes with their ligands $[27,32,45,57]$ or antagonists $[36,55]$ permitted not only the identification of properties common for all TLRs but also suggested a common model for activation of these receptors $[26,31]$. The binding of a ligand induces local subtle changes of the receptor conformation allowing for its dimerization, which, with the exception of the TLR1-TLR2 and TLR2-TLR6 complexes, is homodimerization. Despite the differences in the ligand-binding sites between various TLRs, the arising complex has basically the same shape, resembling the letter $\mathrm{M}$, due to bringing together of the ectodomains in the juxtamembrane region. This, in turn, leads to an approximation of the cytoplasmic domains of the two receptors and their arrangement in an orientation which promotes their dimerization. This is accompanied by conformational alterations in the receptor TIR domain, allowing for their association with the TIR domains of adaptor proteins [53].

Recent studies suggest that TLR4 differs in this respect from the other TLRs. The cytoplasmic and transmembrane domains of TLR4 have an intrinsic capacity to dimerize, in contrast to other receptors of the family [56]. The role of the TLR4 ectodomain is to inhibit the interactions until the specific ligand, LPS, is bound. It seems that such a system leads to a much more potent cell activation than that of the other TLRs. Studies on a constitutively active TLR4 in which the extracellular domain 
was substituted by the corresponding domain of CD4 protein have demonstrated that TLR4 induces expression of pro-inflammatory genes with several-hundred-fold higher potency than the remaining receptors of the family [17]. Since septic shock, a potential effect of infection with Gram-negative bacteria, poses a serious threat to patients of intensive care units, both TLR4 and the mechanisms of its activation by LPS are particularly intensely studied by groups investigating the mechanisms of innate immune responses.

\section{TLR4 TRIGGERS INFLAMMATORY RESPONSE IN REACTION TO LPS}

\section{RECOGNITION OF LPS BY TLR4}

Lipopolysaccharides are a group of compounds with a similar structure, which constitute the main component of the outer membrane of Gram-negative bacteria [69]. Due to their crucial role in the maintenance of the bacterial cell structure, the LPS structure is highly conserved in evolution, providing a model example of PAMP. LPS strongly activates cells of the immune system, inducing an inflammatory response. An excessive reaction to LPS and presence of high LPS concentrations may lead to a systemic inflammatory reaction termed sepsis [65]. Severe sepsis is linked to life-threatening multiple organ dysfunction and the subsequent refractory hypotension provides grounds for diagnosis of septic shock, which in over $30 \%$ of cases leads to the patient's death.

The structure of LPS isolated from several types of bacteria and its modifications have been revealed in detail and described in earlier reviews [11, 60, 62]. LPS consists of three components: the polysaccharide chain termed the $\mathrm{O}$ antigen, the core and lipid A which anchors the LPS molecule in the outer membrane of Gram-negative bacteria. The greatest variability is observed in the composition and number of sugar residues forming the $\mathrm{O}$ antigen. Over 60 types of sugar residues and 30 non-sugar elements modifying them have been identified as components of the $\mathrm{O}$ antigen. The most frequently encountered sugars include hexoses such as glucose, fructose, galactose, mannose and rhamnose. In certain species of bacteria or in specific growth conditions the O-specific chain may be absent or expressed in a truncated form. This results in a „rough" phenotype of bacterial colonies in contrast to the typical „smooth" colonies. It is usually considered that the length and sugar composition of he O antigen do not affect the biological activity of LPS and its high variability make it unlikely to provide an easy target for PRRs. Nevertheless, an absence of the O-specific chain modulates the process of LPS recognition by cells of the immune system, as noted in cases of LPS originating from Brucella 
$s p$. and Salmonella sp. and also from Escherichia coli $[8,25,63]$. Rough LPS, in contrast to LPS with a complete O-specific chain, activates certain cell reactions without an involvement of the CD14 protein $[25,21]$.

The core of the LPS molecule is also an oligosaccharide and contains a residue of 3-deoxy-D-manno-octulosonic acid (KDO) linked to the proximal glucosamine residue of lipid A. The KDO is usually substituted by subsequent residues of KDO and by residues of L-glycero-D-mannoheptose (Hep). The set of 1-3 KDO and 2 or 3 Hep constitutes the so called ,inner core" to which three hexoses are typically attached (the „outer core”). The structure of this part is significantly less variable than that of the $\mathrm{O}$ antigen but may still contain various modifications. The core oligosaccharide is thought to be insignificant for the full biological activity of LPS but may exert some modulatory effect [11].

Lipid A is the most evolutionarily conserved part of LPS. It is the part recognised by receptors of the immune system and, thus, it is responsible for the pro-inflammatory activity of LPS [62]. The basal structure of lipid A is formed by two glucosamine molecules linked by a $\beta-1,6$ glycosidic bond and to this sugar skeleton, 4 to 7 fatty acid residues (depending on bacterial species) of a variable length are attached. The number and length of the fatty acid residues are the main source of lipid A heterogeneity among bacteria and also are the most significant factor affecting the biological activity of LPS. The presence of six chains, each comprising 12 to 14 carbon atoms, affords maximal immune cell activation while addition or removal of just a single chain reduces the pro-inflammatory activity of LPS by over two orders of magnitude [11, 62]. Another factor which determines the ability of LPS to activate the immune system involves phosphorylation of lipid A glycosamines [62]. These two structural properties determine the affinity with which LPS binds MD-2 and TLR4 proteins.

The recognition of LPS is a multi-stage process and it requires co-operation of a number of proteins, the LPS-binding protein (LBP), the CD14 protein and the TLR4/MD-2 complex. LBP is a $60-\mathrm{kDa}$ glycoprotein which participates in detaching LPS monomers from micelles or directly from the bacterial outer membrane and allows for their transport to the CD14 protein. The closely related and structurally similar bactericidal/permeability increasing (BPI) protein binds LPS with a much higher affinity but does not transfer it to CD14. BPI is postulated to be important in LPS detoxification [79]. LBP and CD14 allow for concentration of LPS molecules before transferring them to the TLR4/MD-2 complex assuring a pronounced and rapid cellular response even to low concentrations of LPS (1 $\mathrm{ng} / \mathrm{ml}$ and even lower) in the environment.

CD14 is a $56-\mathrm{kDa}$ protein containing LRR repeats in its extracellular part, in which it resembles TLRs. CD14 forms homodimers and in such a form binds LPS through a hydrophobic pocket located at the outer surface of the dimer [49, 37]. CD14 contains no transmembrane domain and is incorporated in the outer leaflet of 
the plasma membrane via a glycosylphosphatidylinositol (GPI) anchor attached to the $\mathrm{C}$-terminus of the protein. The extracellular phospholipase $\mathrm{C}$ may hydrolyse the ester bond linking the acyl group which anchors CD14 in the plasma membrane with the remaining fragment of the GPI anchor. This leads to a release of CD14 in its soluble form (sCD14), which allows for recognition of LPS by cells devoid of CD14 in the membrane-bound form (mCD14). The presence of the GPI anchor in the membrane form of CD14 points to the association of the protein with so called lipid rafts. These are plasma membrane microdomains enriched in sphingolipids and cholesterol, distinguished from the surrounding more liquid regions of the plasma membrane containing mainly glycerophospholipids. Current views on the raft structure assume that they are dynamic, nano-scale accumulations of lipids and few proteins which during activation of immunoreceptors such as $\mathrm{T}$ cell receptor form more stable platforms facilitating the arrangement of protein complexes taking part in signal transduction [44]. The anchoring of CD14 in lipid rafts suggests the existence of a similar mechanism in the assembly of the CD14/TLR4/ MD-2 signalling complex [71]. Of particular interest are experimental data which confirm the assumption, originating from recent investigations based on manipulation of the lipid composition of the rafts in the plasma membrane: enrichment of the plasma membrane in cholesterol due to a mutation of ABCA1 transporter or incorporation of saturated fatty acids to the membrane induced association of TLR4 with the raft fraction, dimerization of the receptor and expression of genes for pro-inflammatory cytokines $[80,87]$.

Commonly, CD14 and LBP together are assumed to play a role of sensors which effectively capture LPS and transfer it to the TLR4/MD-2 complex. However, CD14 may also have other functions in the process of cell stimulation by LPS. In macrophages and dendritic cells isolated from mice devoid of CD14 protein one of two signalling pathways triggered by TLR4 was found to be impaired (see below). The impaired pathway is dependent on the involvement of TRIF adaptor protein, is linked to endocytosis of activated TLR4 and leads to production of type I interferons [85]. CD14 participates also in LPS internalization by cells along a pathway which fails to activate them but allows for removal of LPS from the environment and for its intracellular detoxification [9].

Within the pathway which activates the pro-inflammatory response of cells, the transfer of LPS from CD14 to the TLR4/MD-2 complex represents the last stage of recognition of the molecule and begins the signal transduction process. MD-2 has a hydrophobic cavity surrounded by two $\beta$-sheets, which forms a broad pocket binding the lipid part of the LPS molecule [55]. MD-2 protein is linked to the outer surface of TLR4 by hydrogen and ionic bonds. The binding of LPS to the MD-2 pocket results in formation of a complex consisting of two TLR4/MD-2 heterodimers (fig. 4). In this process the LPS molecule plays a role of a bridge in the case of the optimal LPS structure containing six fatty acid residues, of which five fill the 
MD-2 pocket while the sixth is partially uncovered and interacts with the hydrophobic pocket of the neighbouring TLR4 molecule. The linkage is additionally stabilised by two phosphate groups of lipid A which form ionic and hydrogen bonds with lysine and arginine residues on the surface of the neighbouring TLR4 and MD-2 molecules [57]. Despite their proximity, direct interactions between the two TLR4 molecules remain weak. This model explains the significance of the role played by lipid A structural modifications for biological activity of LPS, since an absence of the phosphate groups or deviations from the optimum number of fatty acid residues do not allow the formation of a stable signalling complex.

TLR4 was not known to initiate signalling cascades in response to LPS until 1998 [59]. Earlier studies pointed to an involvement in this process of other receptors, such as integrins CD11/CD18b, complement regulatory protein CD55, and scavenger receptors [12]. Studies conducted in the recent decade, based on an analysis of fluorescence resonance energy transfer between labelled proteins and on biochemical analysis of cellular fractions have demonstrated that LPS-induced cell activation leads to formation of complexes in the plasma membrane including

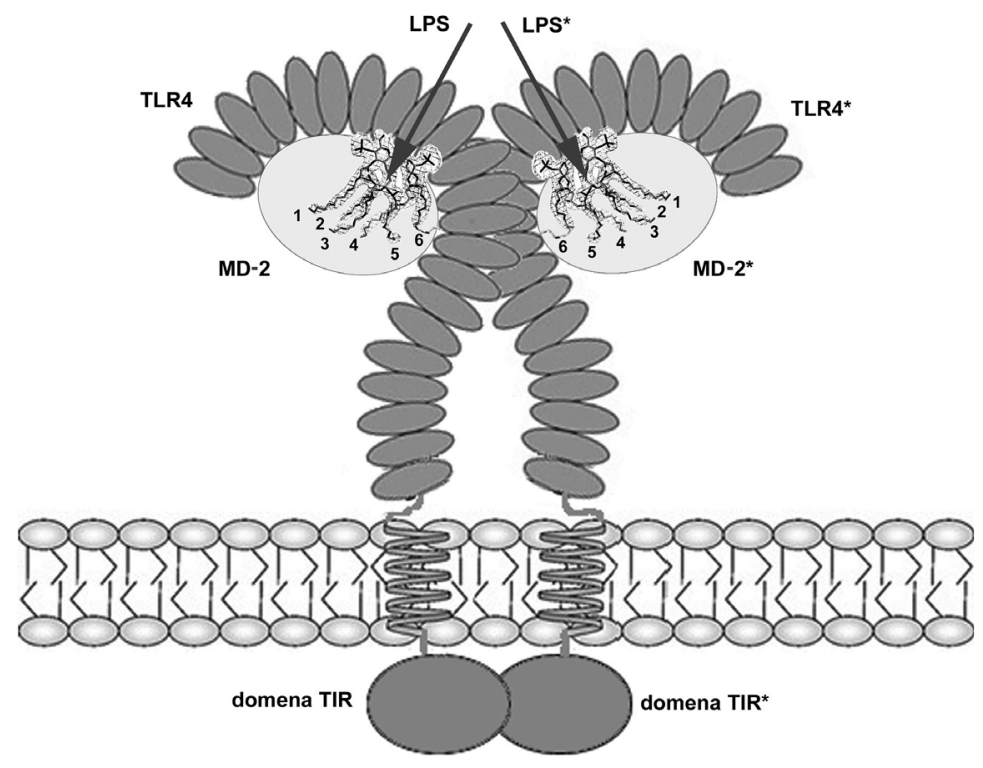

FIGURE 4. Structure of TLR4/MD-2 complex after LPS binding. Five of six fatty acid residues of LPS molecule are buried in the hydrophobic pocket of MD-2 while the sixth residue interacts with a hydrophobic fragment of an adjacent TLR4 molecule. This interaction induces dimerization of TLR4 which brings into proximity cytoplasmic signalling TIR domains of the two TLR4 molecules, facilitating their interactions with adaptor proteins. The complex of two TLR4/MD-2 heterodimers with LPS bound resembles letter M. R-LPS is shown devoid of the O-specific chain. Based on [55-57] 
a number of proteins accompanying CD14 and TLR4 such as heat shock proteins, CD55 and the chemokine receptor, CXCR4 [72]. Formation of the protein complexes in response to LPS may be facilitated by accumulation of the proteins within the earlier-mentioned lipid rafts. Selection of proteins forming such complexes reflects the structural properties of LPS and can modulate the LPS-induced signalling pathways [73]. Studies on the mechanisms of LPS-induced cell stimulation are currently dominated by investigations of the role of TLR4 in this process. Nevertheless, research interest persists regarding the role of scavenger receptors in inflammatory response and current studies uncover a complex pattern of their involvement in the cellular reactions to LPS [28]. Data are available that indicate that members of the class B scavenger receptor family, such as CD36 and SR-B1/Cla-1, induce production of pro-inflammatory cytokines following activation by LPS, independently of TLR4 involvement [2, 40]. In turn, a class A scavenger receptor, SR-A1, seems to play a protective role against excessive cell stimulation by LPS. This reflects the ability of the receptor to internalize high doses of LPS using a pathway which does not induce synthesis of pro-inflammatory cytokines but leads to detoxification of LPS $[19,51]$.

\section{SIGNALLING PATHWAYS OF TLR4}

TLR4 is the only member of the TLR family that interacts with four adaptor proteins containing TIR domains initiating two signalling pathways, the MyD88- and TRIF-dependent one (fig. 5). Interaction of the MyD88 protein with TLR4 occurs with an involvement of an additional adaptor, TIRAP/Mal protein [20]. Apart from the TIR domain allowing for the interaction of MyD88 with TLR4 and TIRAP protein, MyD88 contains in its carboxy-terminal fragment the so called death domain (DD) through which MyD88 interacts with the IRAK4 and IRAK1 kinases (the latter may be substituted by IRAK2 kinase). Crystallographic analyses have shown that six to eight molecules of MyD88 plus four IRAK4 and four IRAK2 (or IRAK1) kinases associate in a hierarchic manner, forming a signalling complex termed the myddosome $[15,43]$. The formation of the myddosome assures close proximity of the IRAK kinases, allowing for phosphorylation of IRAK1 (or IRAK2) kinases by IRAK4. Following the phosphorylation, IRAK1 dissociates from the receptor complex and interacts with TRAF6 (Tumour necrosis factor Receptor Associated Factor $6)$ which functions as an E3 ubiquitin ligase and activates the subsequent signalling kinase TAK1 (Transforming growth factor $\beta$-Associated Kinase-1) [83]. The TAK1 kinase activated in this manner is capable of initiating IKK (ІқВ kinase) and MAPK (mitogen-activated kinase) pathways. This ultimately leads to expression of pro-inflammatory cytokine genes using two transcription factors, $\mathrm{NF}_{\mathrm{B}} \mathrm{B}$ and $\mathrm{AP}-1$. The main pro-inflammatory cytokine produced along the pathway is TNF- $\alpha$ [34]. 


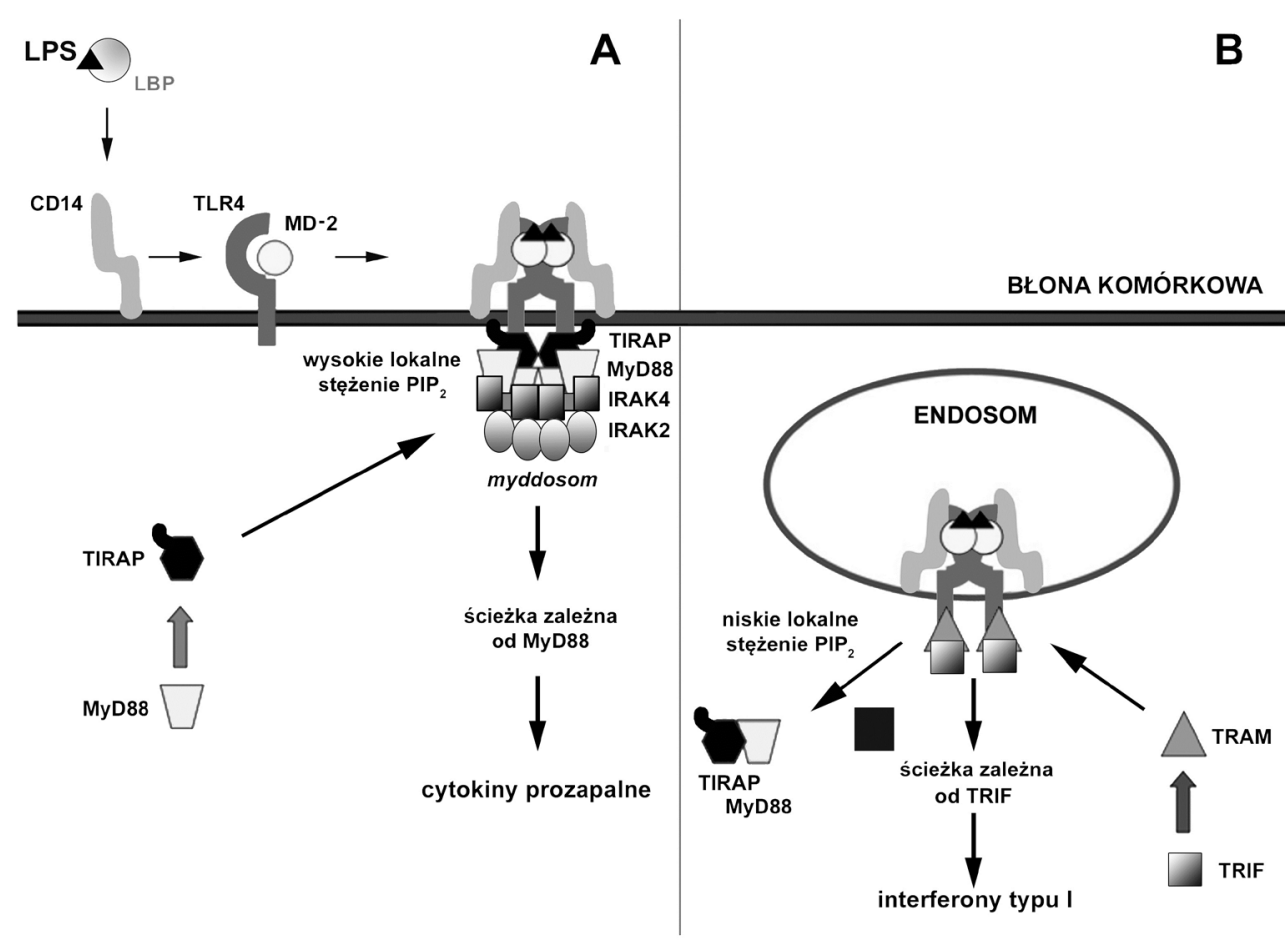

FIGURE 5. Sequential activation of TLR4 signalling pathways dependent on its internalization. A Binding of LPS leads to an assembly of a complex containing CD14, TLR4 and MD-2. A high local concentration of $\mathrm{PI}(4,5) \mathrm{P}_{2}$ allows for binding of the pleckstrin homology domain of TIRAP adaptor protein. On the other hand, the TIR domains of TIRAP molecules interact with TIR domains of TLR4 and MyD88 protein. To the complex, four IRAK4 kinases and four IRAK2 kinases bind via DD domains present also in MyD88. A myddosome is assembled, triggering MyD88-dependent signalling pathway. Between six and eight molecules of MyD88 have been found in the myddosome suggesting a possibility of the assembly of higher-order clusters of activated TLR4 [15, 43]. B - After internalization of TLR4, the concentration of $\mathrm{PI}(4,5) \mathrm{P}_{2}$ in the endosome membrane decreases, leading to dissociation of TIRAP-MyD88 complexes. Adaptor proteins TRAM and TRIF bind to TIR domains of TLR4 triggering the signalling pathway independent of MyD88. Based on [29, 34]

The signalling pathway dependent on the MyD88 adaptor protein is typical for almost all TLRs except TLR3 (fig. 2). On the other hand, the other signalling pathway, dependent on the TRIF adaptor protein, can be initiated only by TLR 4 and TLR3. In order to be recruited, TRIF requires another adaptor protein, TRAM, which mediates its interaction with TLR4 [82]. The TRIF protein, together with a group of accessory proteins, interact with the TBK1 (TANK Binding Kinase-1) and IKKi kinases which phosphorylate transcription factors IRF3 and, to a lower extent, IRF7 [13]. Inactive forms of the IRFs are present in the cytoplasm but following phosphorylation in their C-terminal regions they undergo homodimerization 
and translocation to the nucleus, where they activate transcription of genes coding for type I interferons. Along the TRIF-dependent pathway, apart from phosphorylation of IRF3 and IRF7, also the NFқB transcription factor becomes activated. The TRIF -dependent pathway is suggested to be responsible for late-phase activation of $\mathrm{NF}_{k} \mathrm{~B}$, while the MyD88-dependent pathway leads to early, rapid activation of NFқB directly after LPS binding. Nevertheless, signal transduction along both pathways is required for a full activation of pro-inflammatory cytokine production in response to LPS [34].

An interesting modification of the pathway was detected in the case of TLR7 and TLR9 of dendritic cells, which employ MyD88 for induction of type I interferon synthesis in response to viral infection. In this case the multi-molecular enzymatic complex formed around MyD88 allows for phosphorylation of the IRF7 transcription factor by IRAK1 and IKK $\alpha$ kinases, which induces its translocation to the nucleus and induction of expression of genes coding for type I interferons [33].

For several years TLR4 was assumed to trigger both signalling pathways as a plasma membrane protein. This was regarded a unique property of TLR4 since all other PRRs (including the remaining TLRs, see fig. 2) capable of activating interferon production initiate signal transduction from membranes of intracellular organelles or from the cytoplasm. An alternative model of activation of signalling pathways by TLR4 has been proposed by Kagan and collaborators [29, 30]. This model assumes that the activation of the signalling pathways dependent on MyD88 and on TRIF is temporally and spatially separated (fig. 5). According to the suggested model the membrane receptor complex consisting of CD14 and TLR4/MD-2 is assembled in plasma membrane regions rich in phosphatidylinositol-(4,5)-bisphosphate $\left[\left(\mathrm{PI}(4,5) \mathrm{P}_{2}\right]\right.$. The presence of a $\mathrm{PI}(4,5) \mathrm{P}_{2}$-binding domain (so called pleckstrin homology domain) in the TIRAP adaptor protein allows for its association with the plasma membrane which, in turn, promotes interaction of the TIR domains of this protein, MyD88 and TLR4. Activation of TRIF-dependent pathway is followed by internalization of TLR4. In the membrane of the nascent endosome the concentration of PI(4,5) $\mathrm{P}_{2}$ drops significantly which results in dissociation of MyD88-TIRAP from TLR4. This, in turn, allows for association of the second set of adaptor proteins, TRAM and TRIF, and activation of the TRIF-dependent pathway [29]. It is worth noting that TRAM protein is devoid of the $\mathrm{PI}(4,5) \mathrm{P}_{2}$-binding domain and, therefore, does not interact with the plasma membrane and does not compete with MyD88 for the binding of the TIR domains of TLR4 [30]. Thus, the TIRAP and TRAM proteins play a role of ,sorting adaptors”, securing generation of signalling cascades from distinct cellular compartments.

This model leaves unresolved the questions of factors controlling the internalization of TLR4 and the endocytosis pathway along which it may take place. Some data point to an involvement of dynamin and clathrin in this process, which suggests endocytosis of TLR4 by a classical pathway, within clathrin-coated vesicles, similarly as in the case of transferrin receptor. At first the data were based on a mi- 
croscopic analysis of the co-localization of TLR4, LPS and transferrin and on the inhibitory effect exerted by mutated forms of dynamin or clathrin heavy chain on the internalization of TLR4 [22]. Recently, inhibitors of dynamin enzymatic activity and compounds blocking association of clathrin with its accessory proteins were employed. The inhibitors repressed internalization of activated TLR4 and significantly reduced phosphorylation of IRF3 factor and synthesis of interferon $\beta[30$, 76]. On the other hand, a report by Zanoni and co-workers showed that TLR4 is internalized via macropinocytosis which is controlled by the tyrosine kinase Syk, phospholipase $\mathrm{C} \gamma 2$ and by influx of $\mathrm{Ca}^{2+}$ ions [85]. According to that report, the increase in cytoplasmic $\mathrm{Ca}^{2+}$ ion concentration dependent on the activity of phospholipase $\mathrm{C} \gamma 2$ controls activation of IRF3 and expression of its target genes [6]. The primary factor which controls internalization of TLR4 seems to be its co-receptor, the CD14 protein. In macrophages isolated from mice devoid of CD14, internalization of TLR4 and production of interferon $\beta$ were negligible [85], although the mechanisms of such a relationship remain unknown.

TLR4 also appears in the membrane of phagosomes within which Escherichia coli are internalized. This process initiates the TRIF-dependent signalling pathway and synthesis of type I interferons [23]. Authors of that report indicate that TLR4 is transported to the phagosome membrane from early endosomes and that this stage of intracellular TLR4 transport remains under control of a GTPase from the Rab family, Rab11. This notion is additionally supported by other reports, according to which the TLR4/MD-2 complex is constitutively present in endosome membranes and in the Golgi apparatus of macrophages, from which it may be incorporated to the plasma membrane but it can also recognize LPS and initiate both the TRIF- and MyD88-dependent signalling pathways [68, 75].

The endocytic pathway participates also in terminating the activation of TLR4. Following binding of LPS, TLR4 translocates to late endosomes and lysosomes and becomes degraded. This process involves another Rab-family GTPase, the Rab7b protein, which associates with the lysosome membrane. Activity of this protein plays a significant role in promoting degradation of TLR4 and in negative regulation of TLR4 signalling [74]. The negative control of LPS-induced pro-inflammatory response includes also the internalization of high LPS doses described above, dependent on the participation of scavenger receptors [51].

\section{SUMMARY}

Studies on the mechanisms of signal transduction by TLRs conducted within the last 15 years using diverse genetic, crystallographic and immunologic techniques have significantly broadened our understanding of the immune system functioning. Particular attention was focused on TLR4 due to the high mortality of septic shock 
victims. A breakthrough in the field resulted from studies demonstrating the role of endocytosis in activation of one of TLR4 signalling pathways. However, those discoveries failed to translate into practical application of the accumulated knowledge for controlling sepsis, and the fate of drugs designed on the basis of that knowledge, such as the TLR4 antagonist eritoran, remains uncertain (see: http://www.eisai.com/ news/enews201108pdf.pdf). A more complex approach needs to be applied in studies on TLR4 functioning in the context of other receptors and proteins engaged in immune reactions, such as scavenger receptors and more attention should be paid to their interactions. This type studies should provide novel data on the mechanisms of negative regulation of TLR4 signalling, particularly valuable for diminishing the potentially lethal inflammatory reaction.

\section{ACKNOWLEDGEMENTS}

The study was supported by grant N N303 809040 form the National Science Centre

\section{REFERENCES}

[1] Anderson KV, Nüsslein-Volhard C. Information for the dorsal-ventral pattern of the Drosophila embryo is stored as maternal mRNA. Nature 1984; 311: 223-227.

[2] Baranova IN, Kurlander R, Bocharov AV, Vishnyakova TG, Chen Z, Remaley AT, Csako G, PatterSON AP, EGGERMAN TL. Role of human CD36 in bacterial recognition, phagocytosis, and pathogen-induced JNK-mediated signalling. J Immunol 2008; 181: 7147-7156.

[3] Bianchi Me. DAMPs, PAMPs and alarmins: all we need to know about danger. J Leukoc Biol 2007; 81: $1-5$.

[4] Bortoluci KR, Medzhitov R. Control of infection by pyroptosis and autophagy: role of TLR and NLR. Cell Mol Life Sci 2010; 67: 1643-1651.

[5] Carty M, Goodbody R, Schroder M, Stack J, Moynagh PN, Bowie AG. The human adaptor Sarm negatively regulates adaptor protein TRIF-dependent Toll-like receptor signalling. Nat Immunol 2006; 7: 1074-1081.

[6] Chiang CY, Veckman V, Limmer K, David M. Phospholipase $\mathrm{C} \gamma-2$ and intracellular calcium are required for lipopolysaccharide-induced Toll-like receptor 4 (TLR4) endocytosis and interferon regulatory factor 3 (IRF3) activation. J Biol Chem 2012; 287: 3704-3709.

[7] Choe J, Kelker MS, Wilson IA. Crystal structure of human Toll-like receptor 3 (TLR3) ectodomain. Science 2005; 309: 581-585.

[8] Duerr CU, Zenk SF, Chassin C, Pott J, Gutle D, Hensel M, Hornef MW. O-antigen delays lipopolysaccharide recognition and impairs antibacterial host defense in murine intestinal epithelial cells. PLoS Pathog 2009; 5: e1000567.

[9] Dunzendorfer S, Lee HK, Soldau K, Tobias PS. TLR4 is the signalling but not the lipopolysaccharif de uptake receptor. J Immunol 2004; 173: 1166-1170.

[10] ERRIDGe C. Endogenous ligands of TLR2 and TLR4: agonists or assistants? J Leukoc Biol 2010; 87: 989-999. 
[11] Erridge C, Bennett-Guerrero E, Poxton IR. Structure and function of lipopolysaccharides. Microbes Infect 2002; 4: 837-851.

[12] Fenton MJ, Golenbock DT. LPS-binding proteins and receptors. J Leukoc Biol 1998; 64: 25-32.

[13] Fitzgerald Ka, Mcwhirter SM, Faia Kl, Rowe DC, Latz E, Golenbock DT, Coyle AJ, Liao SM, MANIATIS T. IKKE and TBK1 are essential components of the IRF3 signalling pathway. Nat Immunol 2003; 4: 491-496.

[14] Gay NJ, Gangloff M. Structure and function of Toll receptors and their ligands. Annu Rev Biochem 2007; 76: 141-165.

[15] Gay NJ, Gangloff M, O'Neill LA. What the Myddosome structure tells us about the initiation of innate immunity. Trends Immunol 2011; 32: 104-109.

[16] Grygorowicz MA, KozŁowska E. Involvement of TLR receptors recognising pathogens' molecular patterns in modulation of activity manifested by regulatory lymphocytes $\mathrm{T} C \mathrm{CD} 4^{+} \mathrm{CD} 25^{+} \mathrm{FoxP} 3^{+}($in Polish). Post. Mikrobiol 2011; 50: 141-154.

[17] Hasan UA, Dollet S, Vlach J. Differential induction of gene promoter constructs by constitutively active human TLRs. Biochem Biophys Res Commun 2004; 321: 124-131.

[18] Hasan U, Chaffois C, Gaillard C, Saulnier V, Merck E, Tancredi S, Guiet C, Briere F, Vlach J, Lebecque S, Trinchieri G, Bates EE. Human TLR10 is a functional receptor, expressed by B cells and plasmacytoid dendritic cells, which activates gene transcription through MyD88. J Immunol 2005; 174: 2942-2950.

[19] Haworth R, Platt N, Keshav S, Hughes D, Darley E, Suzuki H, Kurihara Y, Kodama T, Gordon S. The macrophage scavenger receptor type $\mathrm{A}$ is expressed by activated macrophages and protects the host against lethal endotoxic shock. J Exp Med 1997; 186: 1431-1439.

[20] Horng T, Barton GM, Medzhitov IR. TIRAP: an adapter molecule in the Toll signalling pathway. Nat Immunol 2001; 2: 835-841.

[21] Huber M, Kalis C, Keck S, Jiang Z, Georgel P, Du X, Shamel L, Sovath S, Mudd S, Beutler B, Galanos C, Freudenberg MA. R-form LPS, the master key to the activation of TLR4/MD-2-positive cells. Eur J Immunol 2006; 36: 701-711.

[22] Husebye H, Halaas O, Stenmark H, Tunheim G, Sandanger O, Bogen B, Brech A, Latz E, Espevik T. Endocytic pathways regulate Toll-like receptor 4 signalling and link innate and adaptive immunity. EMBO J 2006; 25: 683-962.

[23] Husebye H, Aune Mh, Stenvik J, Samstad E, Skjeldal F, Halaas O, Nilsen NJ, Stenmark H, Latz E, Lien E, Mollnes TE, Bakke O, Espevik T. The Rab11a GTPase controls Toll-like receptor 4-induced activation of interferon regulatory factor-3 on phagosomes. Immunity 2010; 33: 583-596.

[24] Imasaki A, Medzhitov R. Regulation of adaptive immunity by the innate immune system. Science 2010; 327: 291-295.

[25] Jiang Z, Georgel P, Du X, Shamel L, Sovath S, Mudd S, Huber M, Kalis S, Keck S, Galanos C, Freudenberg M, Beutler B. CD14 is required for MyD88-independent LPS signalling. Nat Immunol 2005; 6: 565-570.

[26] JIN Ms, LeE Jo. Structures of the toll-like receptor family and its ligand complexes. Immunity 2008; 29: 182-191.

[27] Jin MS, Kim SE, Heo JY, Lee ME, Kim HM, Paik SG, Lee H, Lee JO. Crystal structure of the TLR1TLR2 heterodimer induced by binding of a tri-acylated lipopeptide. Cell 2007; 130: 1071-1082.

[28] Józefowski S. The role of the class A scavenger receptors, SR-A and MARCO, in the immune system. Part 1. The structure of receptors, their ligand binding repertoires and ability to initiate intracellular signalling. Postepy Hig Med Dosw (Online) 2012; 6: 104-119.

[29] Kagan JC, Medzhitov R. Phosphoinositide-mediated adaptor recruitment controls Toll-like receptor signalling. Cell 2006; 125: 943-955.

[30] Kagan JC, Su T, Horng T, Chow A, Akira S, Medzhitov R. TRAM couples endocytosis of Toll-like receptor 4 to the induction of interferon- $\beta$. Nat Immunol 2008; 9: 361-368. 
[31] Kang JY, Lee JO. Structural biology of the Toll-like receptor family. Annu Rev Biochem 2011; 80: 917-941.

[32] Kang JY, Nan X, Jin MS, Youn SJ, Ryu JH, Mah S, Han SH, Lee H, Paik SG, Lee JO. Recognition of lipopeptide patterns by Toll-like receptor 2-Toll-like receptor 6 heterodimer. Immunity 2009; 31: 873-884.

[33] Kawai T, AkiRa S. Toll-like receptor and RIG-I-like receptor signalling. Ann N Y Acad Sci 2008; 1143: $1-20$.

[34] KaWAi T, AkIRA S. The role of pattern-recognition receptors in innate immunity: update on Toll-like receptors. Nat Immunol 2010; 11: 373-384.

[35] Kennedy MN, Mullen Ge, Leifer CA, Lee C, Mazzoni A, Dileepan KN, Segal DM. A complex of soluble MD-2 and lipopolysaccharide serves as an activating ligand for Toll-like receptor 4. J Biol Chem 2004; 279: 34698-34704.

[36] Kim HM, Park BS, Kim Ji, Kim SE, Lee J, Oh SC, Enkhbayar P, Matsushima N, Lee H, Yoo OJ, Lee JO. Crystal structure of the TLR4-MD-2 complex with bound endotoxin antagonist Eritoran. Cell 2007; 130: 906-917.

[37] Kim JI, Lee CJ, Jin MS, Lee CH, Paik SG, Lee H, Lee JO. Crystal structure of CD14 and its implications for lipopolysaccharide signalling. J Biol Chem 2005; 280: 11347-11351.

[38] Kobe B, Kajava AV. The leucine-rich repeat as a protein recognition motif. Curr Opin Struct Biol 2001; 11: 725-732.

[39] Kumar H, Kawai T, Akira S. Pathogen recognition by the innate immune system. Int Rev Immunol 2011; 30: 16-34.

[40] Leelahavanichkul A, Bocharov AV, Kurlander R, Baranova IN, Vishnyakova TG, Souza AC, Hu X, Doi K, Vaisman B, Amar M, Sviridov D, Chen Z, Remaley AT, Csako G, Patterson AP, Yuen PS, StAR RA, EgGerman TL. Class B scavenger receptor types I and II and CD36 targeting improves sepsis survival and acute outcomes in mice. J Immunol 2012; 188: 2749-2758.

[41] Lemaitre B, Nicolas E, Michaut L, Reichhart JM, Hoffmann JA. The dorsoventral regulatory gene cassette spatzle/Toll/cactus controls the potent antifungal response in Drosophila adults. Cell 1996; 86: $973-983$.

[42] Leulier F, Lemaitre B. Toll-like receptors - taking an evolutionary approach. Nat Rev Genet 2008; 9: 165-178.

[43] Lin SC, Lo YC, Wu H. Helical assembly in the MyD88-IRAK4-IRAK2 complex in TLR/IL-1R signalling. Nature 2010; 465: 885-890.

[44] Lingwood D, Simons K. Lipid rafts as a membrane-organizing principle. Science 2010; 327: 46-50.

[45] Liu L, Botos I, Wang Y, Leonard JN, Shiloach J, Segal DM, Davies DR. Structural basis of toll-like receptor 3 signalling with double-stranded RNA. Science 2008; 320: 379-381.

[46] MAcLeod H, WETZLER LM. T cell activation by TLRs: a role for TLRs in the adaptive immune response. Sci STKE 2007; 2007(402): e48.

[47] Matsushima N, Tanaka T, Enkhbayar P, Mikami T, Taga M, Yamada K, Kuroki K. Comparative sequence analysis of leucine-rich repeats (LRRs) within vertebrate toll-like receptors. BMC Genomics 2007; 8: 124.

[48] Medzhitov R, Preston-Hurlburt P, Janeway CA Jr. A human homologue of the Drosophila Toll protein signals activation of adaptive immunity. Nature 1997; 388: 394-397.

[49] Meng J, Parroche P, Golenbock DT, Mcknight CJ. The differential impact of disulfide bonds and N-linked glycosylation on the stability and function of CD14. J Biol Chem 2008; 283: 3376-33784.

[50] Mishra BB, Gundra UM, Teale JM. Expression and distribution of Toll-like receptors 11-13 in the brain during murine neurocysticercosis. J Neuroinflammation 2008; 5: 53. 
[51] Mukhopadhyay S,Varin A, Chen Y, Liu B, Tryggvason K, Gordon S. SRA/MarCO-mediated ligand delivery enhances intracellular TLR and NLR function, but ligand scavenging from cell surface limits TLR4 response to pathogens. Blood 2011; 117: 1319-1328.

[52] Nagai Y Akashi S, Nagafuku M, Ogata M, Imakura Y, Akira S, Kitamura T, Kosugi A, Kimoto M, MIYAKE K. Essential role of MD-2 in LPS responsiveness and TLR4 distribution. Nat Immunol 2002; 3: $667-672$.

[53] Nunez Miguel R, Wong J, Westoll JF, Brooks HJ, O’neill LA, Gay NJ, Bryant CE, Monie TP. A dimer of the Toll-like receptor 4 cytoplasmic domain provides a specific scaffold for the recruitment of signalling adaptor proteins. PLoS One 2007; 2: e788.

[54] Nyman T, Stenmark P, Flodin S, Johansson I, Hammarstrom M, Nordlund P. The crystal structure of the human Toll-like receptor 10 cytoplasmic domain reveals a putative signalling dimer. J Biol Chem 2008; 283: 11861-11865.

[55] Ohto U, Fukase K, Miyake K, Satow Y. Crystal structures of human MD-2 and its complex with antiendotoxic lipid IVa. Science 2007; 316: 1632-1633.

[56] Panter G, Jerala R. The ectodomain of the Toll-like receptor 4 prevents constitutive receptor activation. J Biol Chem 2011; 286: 23334-13344.

[57] Park BS, Song DH, Kim HM, Choi BS, Lee H, Lee JO. The structural basis of lipopolysaccharide recognition by the TLR4-MD-2 complex. Nature 2009; 458: 1191-1195.

[58] Pifer R, Benson A, Sturge CR, Yarovinsky F. UNC93B1 is essential for TLR11 activation and IL-12-dependent host resistance to Toxoplasma gondii. J Biol Chem 2011; 286: 3307-3314.

[59] Poltorak A, He X, Smirnova I, Liu MY, Van Huffel C, Du X, Birdwell D, Alejos E, Silva M, Galanos C, Freudenberg M, Ricciardi Castagnoli P, Layton B, Beutler B. Defective LPS signalling in $\mathrm{C} 3 \mathrm{H} / \mathrm{HeJ}$ and C57BL/10ScCr mice: mutations in Tlr4 gene. Science 1998; 282: 2085-2088.

[60] Raetz CR, Whitfield C. Lipopolysaccharide endotoxins. Annu Rev Biochem 2002; 71: 635-700.

[61] Resman N, Vasl J, Oblak A, Pristovsek P, Gioannini TL, Weiss JP, Jerala R. Essential roles of hydrophobic residues in both MD-2 and toll-like receptor 4 in activation by endotoxin. J Biol Chem 2009; 284: $15052-15060$.

[62] Rietschel ET, Kirikae T, Schade FU, Mamat U, Schmidt G, Loppnow H, Ulmer AJ, Zahringer U, Seydel U, Di Padova F, Schreier M, Brade H. Bacterial endotoxin: molecular relationships of structure to activity and function. FASEB J 1994; 8: 217-225.

[63] Rittig MG, Kaufmann A, Robins A, Shaw B, Sprenger H, Gemsa D, Foulongne V, Rouot B, Dornand J. Smooth and rough lipopolysaccharide phenotypes of Brucella induce different intracellular trafficking and cytokine/chemokine release in human monocytes. J Leukoc Biol 2003; 74: 1045-1055.

[64] Rolls A, Shechter R, London A, Ziv Y, Ronen A, Levy R, Schwartz M. Toll-like receptors modulate adult hippocampal neurogenesis. Nat Cell Biol 2007; 9: 1081-1088.

[65] Salomao R, Brunialti MK, Rapozo MM, Baggio-Zappia GL, Galanos C, Freudenberg M. Bacterial sensing, cell signalling, and modulation of the immune response during sepsis. Shock 2012; 38: 227-242.

[66] Satoh T, Kato H, Kumagai Y, Yoneyama M, Sato S, Matsushita K, Tsujimura T, Fujita T, Akira S, TAKEUCHI O. LGP2 is a positive regulator of RIG-I and MDA5-mediated antiviral responses. Proc Natl Acad Sci USA 2010. 107: 1512-517.

[67] Schnare M, Barton GM, Holt aC, Takeda K, Akira S, Medzhitov R. Toll-like receptors control activation of adaptive immune responses. Nat Immunol 2001; 2: 947-950.

[68] Shibata T, Motoi Y, Tanimura N, Yamakawa N, Akashi-Takamura S, Miyake K. Intracellular TLR4/ MD-2 in macrophages senses Gram-negative bacteria and induces a unique set of LPS-dependent genes. Int Immunol 2011; 23: 503-510. 
[69] Silhavy TJ, Kahne D, Walker S. The bacterial cell envelope. Cold Spring Harb Perspect Biol 2010; 2: a000414.

[70] Sun J, Duffy Ke, Ranjith-Kumar CT, Xiong J, Lamb RJ, Santos J, Masarapu H, Cunningham M, Holzenburg A, Sarisky RT, Mbow ML, Kao C. Structural and functional analyses of the human Tol1-like receptor 3. Role of glycosylation. J Biol Chem 2006; 281: 11144-11151.

[71] Triantafilou M, Morath S, Mackie A, Hartung K, Triantafilou K. Lateral diffusion of Toll-like receptors reveals that they are transiently confined within lipid rafts on the plasma membrane. J Cell Sci 2004; 117: 4007-4014.

[72] Triantafilou M, Lepper PM, Briault CD, Ahmed MA, Dmochowski JM, Schumann C, Triantafilou $\mathrm{K}$. Chemokine receptor 4 (CXCR4) is part of the lipopolysaccharide "sensing apparatus". Eur J Immunol 2008; 38: 192-203.

[73] Triantafilou M, Lepper PM, Olden R, Dias IS, Triantafilou K. Location, location, location: Is membrane partitioning everything when it comes to innate immune activation? Mediators Inflamm 2011; 2011: 186093.

[74] Wang Y, Chen T, Han C, He D, Liu H, An H, Cai Z, Cao X. Lysosome-associated small Rab GTPase Rab7b negatively regulates TLR4 signalling in macrophages by promoting lysosomal degradation of TLR4. Blood 2007; 110: 962-971.

[75] Wang D, Lou J, Ouyang C, Chen W, Liu Y, Liu X, Cao X, Wang J, Lu L. Ras-related protein Rab10 facilitates TLR4 signalling by promoting replenishment of TLR4 onto the plasma membrane. Proc Natl Acad Sci USA 2010; 107: 13806-13811.

[76] Wang Y, Yang Y, Liu X, Wang N, Cao H, Lu Y, Zhou H, Zheng J. Inhibition of clathrin/dynamin-dependent internalization interferes with LPS-mediated TRAM-TRIF-dependent signalling pathway. Cell Immunol 2012; 274: 121-129.

[77] Watters TM, Kenny EF, O'NeILl LA. Structure, function and regulation of the Toll/IL-1 receptor adaptor proteins. Immunol Cell Biol 2007; 85: 411-419.

[78] Weber AN, Morse MA, Gay NJ. Four N-linked glycosylation sites in humantoll-like receptor 2 coe operate to direct efficient biosynthesis and secretion. J Biol Chem 2004; 279: 34589-34594.

[79] WeISS J. Bactericidal/permeability-increasing protein (BPI) and lipopolysaccharide- binding protein (LBP): structure, function and regulation in host defence against Gram- negative bacteria. Biochem Soc Trans 2003; 31: 785-790.

[80] Wong SW, Kwon MJ, Choi AM, Kim HP, Nakahira K, Hwang DH. Fatty acids modulate Toll-like receptor 4 activation through regulation of receptor dimerization and recruitment into lipid rafts in a reactive oxygen species-dependent manner. J Biol Chem 2009; 284: 27384-27392.

[81] Xu Y, Tao X, Shen B, Horng T, Medzhitov R, Manley JL, Tong L. Structural basis for signal transduction by the Toll/interleukin-1 receptor domains.Nature, 2000; 408: 111-115.

[82] Yamamoto M, Sato S, Hemmi H, Uematsu S, Hoshino K, Kaisho T, Takeuchi O, Takeda K, Akira S. TRAM is specifically involved in the Toll-like receptor 4-mediated MyD88-independent signalling pathway. Nat Immunol 2003; 4: 144-1150.

[83] Yamamoto M, Оkamoto T, Takeda K, Sato S, Sanjo H, Uematsu S, Saitoh T, Yamamoto N, Sakurai H, Ishit KJ, Yamaoka S, Kawai T, Matsuura Y, Takeuchi O, Akira S. Key function for the Ubc13 E2 ubiquitin-conjugating enzyme in immune receptor signalling. Nat Immunol 2006; 7: 962-970.

[84] Yang H, Hreggvidsdottir HS, Palmblad K, Wang H, Ochani M, Li J, Lu B, Chavan S, Rosas-Ballina M, Al-Abed Y, Akira S, Bierhaus A, Erladsson-Harris H, Abdersson U, Tracey KJ. A critical cysteine is required for HMGB1 binding to Toll-like receptor 4 and activation of macrophage cytokine release. Proc Natl Acad Sci USA 2010; 107: 11942-11947. 
[85] Zanoni I, Ostuni R, Marek LR, Barresi S, Barbalat R, Barton GM, Granucci F, Kagan JC. CD14 controls the LPS-induced endocytosis of Toll-like receptor 4. Cell 2011; 147: 868-880.

[86] Zhang D, Zhang G, Hayden MS, Greenblatt MB, Bussey C, Flavell RA, Ghosh IS. A toll-like receptor that prevents infection by uropathogenic bacteria.Science 2004; 303: 1522-1526.

[87] Zhu X, Owen JS, Wilson MD, Li H, Griffiths GL, Thomas MJ, Hiltbold EM, Fessler MB, Parks JS. Macrophage ABCA1 reduces MyD88-dependent Toll-like receptor trafficking to lipid rafts by reduction of lipid raft cholesterol. J Lipid Res 2010; 51: 3196-3206.

Editor - Barbara Ptytycz

Katarzyna Kwiatkowska

Department of Cell Biology

Nencki Institute of Experimental Biology

3 Pasteur Street

02-093 Warsaw

tel: 225892463

e-mail:k.kwiatkowska@nencki.gov.pl 